\title{
Effect of Progestin-Dominant Combined Oral Contraception on Uterine Fibroid Development
}

\author{
Driak D*, Sehnal B, Neumannova $H$, Hurt K, \\ Vasicka I and Halaska $M$ \\ Department of Gynaecology and Obstetrics, Charles \\ University in Prague, Czech Republic, Europe \\ *Corresponding author: Daniel Driák, Department \\ of Gynaecology and Obstetrics, Charles University in \\ Prague, First Faculty of Medicine and Hospital Bulovka, \\ Prague, Czech Republic, Europe
}

Received: October 04, 2017; Accepted: November 22, 2017; Published: November 29, 2017

\begin{abstract}
Background: Combined oral contraception blocks the endogenous ovarian steroid secretion that stabilizes the hormonal environment. Monophasic, progestin-dominant, contraceptives may lead to regression of estrogendependent diseases such as uterine fibroids and endometriosis.

Study Design: The purpose of our randomized, controlled, single-blind, prospective observational study was to document the development of uterine fibroids exposed to the influence of combined oral contraceptive with progestin dominancy. A total of 129 patients were randomly divided into 2 groups: 97 women with one or multiple uterine fibroids treated conservatively with monophasic hormonal pills containing $20 \mathrm{mcg}$ of ethinyl estradiol and $75 \mathrm{mcg}$ of gestodene were observed for a period of 2 to 4 years, and 32 women in the control group.
\end{abstract}

Results: In 75 patients ( $77.3 \%$ vs. $31.2 \%$ of untreated controls), regression or no growth was registered. In 22 patients (22.7\%), the size of myoma increased, menstruation irregularity and heavy bleeding continued or worsened. In untreated controls, 22 out of 32 patients $(68.8 \%)$ continued suffering from dysfunctional bleeding and abdominal pain associated with uterine fibroids and requested another mode of therapy. The changes in the volume of myomas between the experimental and control group were statistically significant $(p=$ 0.044).

Conclusion: Use of low-dose hormonal contraceptives with progestin dominancy can lead to significant reduction in myoma volume.

Keywords: Uterine fibroids; Myoma; Contraception; Progestin dominancy; Growth; Regression

\section{Introduction}

Combined hormonal (estrogen-progestin) contraception offers many non-contraceptive benefits. The favorable protective and therapeutical effects comprising some gynecological as well as nongynecological disorders are evidence-based and commonly used in clinical practice. Among the most important non-contraceptive benefits are the reduction of the risk of ovarian, endometrial and colorectal cancer, treatment of menorrhagia, dysmenorrhea, premenstrual syndrome, acne vulgaris, endometriosis and uterine fibroids [1]. Otherwise, other hormonal contraceptive methods offering some benefits are also available (progestin-only pills and the intrauterine system continuously releasing levonorgestrel) [2,3].

Uterine fibroid (leiomyoma) is a benign, mesenchymal tumor mostly originating in smooth muscle of the uterus. It is the most frequent gynecological tumor that occurs in more than $20 \%$ of women above 30 years of age and in 30-40\% of women between $40-50$ years. Leiomyoma is rare before menarche, spontaneously diminishes, and may even fade after menopause. In the Czech Republic as well as in other developed countries, leiomyoma is the most frequent indication for hysterectomy and $70-80 \%$ of hysterectomies are performed for myoma and menorrhagia [4].

Despite its high incidence, the causal etiology of leiomyoma remains unknown. There is an incontestable process of at least two steps. In the beginning, somatic mutation of one leiomyocyte occurs, and in cases of multiple fibroids, independently of the others. The genetic predisposition for multiple mutations is suggested, however, the specific genes have not been identified. The second step comprises cellular proliferation under hyperestrogenic conditions. The internal milieu of myoma is hyperestrogenous with high aromatase activity and a high concentration of estrogen receptors. Besides estrogens, the following other growth factors are present: over-expression of Epidermal Growth Factor (EGF) and Insulin-Like Growth Factor I and II (IGFs). Abnormal vascularization is influenced by vascular endothelial growth factor. In addition, an increased concentration of a strong inhibitor of apoptosis, B-cell lymphoma 2 (bcl-2), is observed [5].

According to number, myomas might be solitary or multiple. With respect to localization, myoma can be divided into intramural, cervical, subserousal, intraligamentous, submucousal and myoma nascens - see Figure 1 [6]. According to histopathology, myomas can be differentiated into: benign myoma (with 2 or less mitoses in 10 microscopic fields), cellular (proliferating, atypical) myoma (2-9 mitoses in 10 microscopic fields), and leiomyosarcoma (with more than 10 mitoses in 10 microscopic fields, cytological atypias and the presence of coagulation necrosis) [7]. 


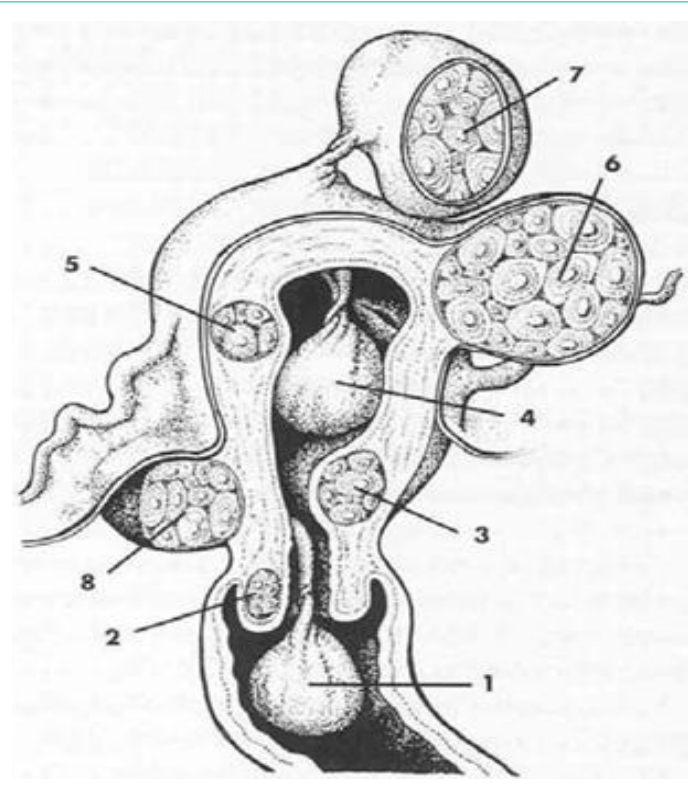

Figure 1: Localization of myomas-with authorized permission of Galen Publishers.

1: Myoma nascens; 2: Cervical myoma; 3: Submucousal myoma; 4:Submucousal pendulating myoma; 5:Intramural myoma; 6: Subserousal myoma; 7: Subserousal pendulating myoma; 8: Intraligamentous myoma.

According to the British study, 35-64\% of women with leiomyomas suffer from dysfunctional uterine bleeding [8]; in some of them resulting in anemia (35\%) [2]. The other symptoms associated with uterine fibroids are abdominal and pelvic pain, dysmenorrhea, dyspareunia, subfebrility, problems arising from pressure on surrounding organs (dysuria, urge incontinence, urine retention, hydronephrosis, constipation, tenesmus), and problems with fertility [4].

Some studies hypothesize that hormonal contraception acts differently on myometrial and on the fibromuscular tissue of leiomyoma. The main benefit may be associated with decreased proliferation and increased apoptosis of the tissues [9].

The aim of this randomized, controlled, single-blind, prospective observational study was to document the changes in uterine fibroids that were exposed to the influence of low-dose combined oral contraceptives with progestin dominancy.

\section{Materials and Methods}

A total of 129 women were enrolled in the study. A total of 97 patients in fertile age suffering from solitary or multiple uterine fibroids were treated conservatively with monophasic Combined Oral Contraception (COC) containing $20 \mathrm{mcg}$ of ethinylestradiol and $75 \mathrm{mcg}$ of gestodene. Fifty-seven women presented with symptoms typically connected with myoma. Dilatation and curretage for malignancy exclusion was performed before initiating hormonal treatment only in cases of metrorrhagia. The COC was administered in a conventional 21 -day regimen with a 7 -day pause.

From the whole study population, the control group was selected by randomization-each fourth was chosen. The group consisted of 32 patients of fertile age with solitary or multiple leiomyomas willing to be treated conservatively. Non-hormonal placebo pills were administered by the same 21-day regimen.

Changes in the growth of uterine myoma and clinical presentation were observed for a period of 2 to 4 years. The ultrasound device Toshiba Nemio SSA-550A with vaginal probe PVM-651VT of 6 $\mathrm{MHz}$ for detection and measurement of fibroids was used. Before beginning the study and at each 3 months following the first week of menstrual bleeding, an ultrasound examination and measurement of fibroids was performed, and the maximal diameter of the myoma was registered.

Statistical analysis was used to evaluate changes over time (MannWhitney Ran Sum test), using Sigma Stat 3.5. $\mathrm{P}<0.05$ was considered statistically significant.

All the enrolled patients had given written informed consent approved by the Ethical Committee of University Hospital Bulovka.

\section{Results}

During the period from 2 to 4 years, solitary or multiple uterine fibroids of $8-65 \mathrm{~mm}$ in diameter with various localizations were evaluated. In 74/97 patients (76.3\%) treated with monophasic COC, regression or no changes in growth were registered, and then were followed-up for 2 years. The patients treated with COC did not have any clinical disorders. Twenty- five patients (25.8\%) with no changes in medication were lost from our records after 2 years, 49 (50.5\%) continued for 4 years wherein on the same medication. In 13/97 patients (13.4\%) under monophasic combined pills, the growth of fibroids as well as problems like menorrhagia, anemia, abdominal pain and dysmenorrhea continued or increased. Main problem in 10 patients was severe uterine bleeding (approximate blood loss of $300 \mathrm{ml}$ ), 3 suffered from abdominal and pelvic pain. They underwent operation: in 3 cases myoma enucleation and in 10 cases laparoscopically assisted vaginal hysterectomy was performed. In 3 of these cases, the lack of effectiveness of the oral contraceptive pills can be explained by poor patient compliance with the daily prescribed dosing regimen. In another patient (1\%) who entered menopause after 1 year, growth of myoma and bleeding ceased, however, despite this fact, she insisted on hysterectomy due to fear of cancer. The histology of leiomyosarcoma was not found in any case. Despite findings of slow growth (in $\mathrm{mm}$ ) without clinical symptomatology in 6 cases and metrorrhagia due to irregular hormonal therapy in 3 cases, all 9 of these patients (9.3\%) refused recommended operative treatment.

Thirty-two patients of the control group were followed-up for 6 months. After 6 months, 8 of 32 women (25\%) refused to undergo further treatment because of irregular bleeding and abdominal pain. Another 14 women (43.8\%) suffering from the same symptoms completed follow-up of 12 months and then left the study. Ten patients $(31.2 \%)$ reached menopause during the time of the study and/or neither clinical disorder nor growth of myoma was recorded.

We found statistically significant $(p=0.044)$ changes in the volume of myomas between the experimental and control group. In the experimental group administered with COC for the period of 2 years, myoma volumes dropped significantly (from a mean value of 35.3 to $30.2 \mathrm{~mm}$ at 2 years, $\mathrm{p}=0.040$ ). The myomas volume in 
the placebo group slightly increased, however the change was not statistically significant (from a mean value of 35.3 to $36.8 \mathrm{~mm}, \mathrm{p}=$ $0.714)$.

\section{Discussion}

The therapy for uterine fibroids is both pharmacological and surgical. The basis of pharmacological therapy is hormonal blockade of endogenous steroidogenesis with Gonadotropin-Releasing Hormone $(\mathrm{GnRH})$ agonists (buserelin, triptorelin, goserelin etc.), combined hormonal contraception with progestin dominancy, progestin-only contraceptives, and intrauterine system continuously releasing levonorgestrel (LNG-IUS). Monophasic combined contraceptives in conventional 21-days as well as in long-cycle treatment, block the endogenous production of steroids and stabilize hormonal concentrations [10]. In comparison with GnRH agonists, contraceptives are less expensive and have minimal metabolic effects. The role of progestin is still controversial. Progesterone stimulates the production of EGF and expression of EGF receptors is stimulated by estrogens. In addition, progesterone stimulates the expression of bcl-2, however, causes inhibition of IGF-I and, by down-regulation of IGF-I receptors blocks the growth of myoma [5]. Norethisterone and other progestins with residual estrogenous activity should not be used because they might stimulate the growth of myomas. A decrease in size of uterine fibroids was observed with treatment of selective progesterone receptor modulators RU-486 (mifepristone). The effect of aromatase inhibitors on myomas is under clinical study. Antigonadotropins (danazol and gestrinone) should not be used due to their severe residual androgenous side effects such as acne, hirsutism, body weight gain, hepatotoxicity. Tamoxifen from the group of selective estrogen receptor modulators by partial estrogenous activity may stimulate the growth of myoma [4].

According to the results of our prospective, controlled, singleblind study on an observed group of women treated with COC with progestin dominancy (containing $20 \mathrm{mcg}$ of ethinylestradiol and $75 \mathrm{mcg}$ of gestodene), a significant number of patients suffering from problems associated with uterine fibroids may otherwise be successfully treated conservatively ( 75 out of $97 ; 77.3 \%$ ). We observed not only diminishing clinical problems associated with uterine fibroids such as menorrhagia, abdominal pain, etc., but also a decrease in size of myomas. In women avoiding operation, conservative therapy with hormonal contraceptives with progestin dominancy might be considered a suitable alternative. In untreated controls, 10 out of 32 patients (31.2\%) did not have disorders associated with uterine fibroids. In 22/97 women (22.7\%), therapy with monophasic contraception failed, in 3 due to poor compliance with daily pil usage. In untreated controls, 22 out of 32 women (68.8\%) suffered from typical problems and requested another mode of therapy.

LNG-IUS suppresses proliferation and induces apoptosis of leiomyocytes. The proliferation of leiomyoma is blocked under minimal concentrations of $10 \mathrm{mcg} / \mathrm{mL}$. The same concentration of LNG significantly decreases the levels of IGF I, bcl-2, survivin, and increases activation of caspase 3 [11]. Sayed et al. [12] documented a $23.1 \%$ failure of LNG-IUS and $37.9 \%$ failure of low-dose COC in 58 patients treated for fibroid-related menorrhagia. In our study, the failure rate of monophasic pills containing $20 \mathrm{mcg}$ of ethinylestradiol and $75 \mathrm{mcg}$ of gestodene was only $22.7 \%$. This may be explained by the use of contraceptives with a very-low dose of ethinylestradiol and a gestodene whose gestational activity in vivo is one of the strongest of all progestins.

In single-blind studies, the subjects do not know whether they are really treated or supplied with placebo without effective compound. However, there are some limitations and risks of single-blind trials such as so called experimenter's bias. The subjects might be consciously or unconsciously influenced by investigators.

Monophasic combined contraception significantly blocks the endogenous production of steroids and stabilizes hormonal concentrations. It might be considered as a suitable alternative for women avoiding operative therapy for various reasons. An important requirement for conservative therapy is periodic regular ultrasound examinations for myoma detection. In cases of sudden abrupt growth, the origin of leiomyosarcoma must be determined, conservative therapy immediately ceased, and operative therapy considered.

\section{References}

1. Huber JC, Bentz EK, Ott J, Tempfer CB. Non-contraceptive benefits of oral contraceptives. Expert Opin Pharmacother. 2008; 9: 2317-2325.

2. Fraser IS. Non-contraceptive health benefits of intrauterine hormonal systems. Contraception. 2010; 82: 396-403.

3. Zapata LB, Whiteman MK, Tepper NK. Intrauterine device use among women with uterine fibroids: a systematic review. Contraception. 2010; 82: 41-55.

4. Tošner J. Analysis of case report study: uterine fibroid. Gynekolog. 2006; 15: 25-33.

5. Kolařík D, Halaška M, Feyereisl J. Repetitorium gynekologie. Praha: Maxdorf. 2008.

6. Citterbart K, Čech E, Donát J. Gynekologie. Praha: Galén. 2001.

7. Giuntoli RL, Bristow RE. Uterine smooth muscle tumors: the benign, the bizarre, and the deadly. Gynekologie po promoci. 2006; 6: 16-23.

8. Overton C, Hargreaves J, Maresh M. A national survey of the complications of endometrial destruction for menstrual disorders: the MISTLETOE study. BJOG. 1997; 104: 1351-1359.

9. Kriplani A, Awasthi D, Kulshrestha V. Efficacy of the levonorgestrel-releasing intrauterine system in uterine leiomyoma. Int J Gynecol Obstet. 2012; 1: 3538.

10. Kuhl H, Birkhäuser M, Mueck A, Neulen J, Thaler C, Kiesel L, et al. Longcycle treatment in oral contraception. Ther Umsch. 2009; 66: 101-108.

11. Xu Q, Qiu L, Zhu L, Luo L, Xu C. Levonorgestrel inhibits proliferation and induces apoptosis in uterine leiomyoma cells. Contraception. 2010; 82: 301308.

12. Sayed GH, Zakherah MS, El-Nahar SA. A randomized clinical trial of a levonorgestrel-releasing intrauterine system and a low-dose combined oral contraceptive for fibroid-related menorrhagia. Int J Gynaecol Obstet. 2011; 11: $126-130$
Austin J Obstet Gynecol - Volume 4 Issue 3 - 2017 Submit your Manuscript | www.austinpublishing group.com Driak et al. () All rights are reserved
Citation: Driak D, Sehnal B, Neumannova H, Hurt K, Vasicka I and Halaska M. Effect of Progestin-Dominant Combined Oral Contraception on Uterine Fibroid Development. Austin J Obstet Gynecol. 2017; 4(3): 1077. 